\title{
Sine-Gordon Model
}

\subsection{The Dawning of Solitons: From the Frenkel-Kontorova Model to the Sine-Gordon Model}

It is traditional to start a discussion of the solitons with a famous story about John Scott Russell, who first observed and described the solitary waves. Many books on solitons begin from this starting point (see, e.g., [310] or [383]). Here, however, we take another route, departing from the Frenkel-Kontorova model introduced in 1938 [155]..$^{1}$ This one-dimensional toy model describes a chain of particles, coupled by the horizontal springs to the nearest neighbors and placed in a periodic potential, which, for example, represents a substrate.

As with every good toy model, it has a lot of other realizations. For example, it can be visualized as a system of two parallel superconducting wires with a Josephson junction in between, or even as a model of the basic functions of DNA (see, e.g., [115]).

Here we consider another mechanical analog of the Frenkel-Kontorova model, which was suggested by Scott in 1969 [352]. This is a chain of identical simple pendulums of length $l$ and mass $m$ separated by distance $a$. The pendulums are oscillating in parallel planes and are elastically coupled through the identical torque springs with their nearest neighbors (see Figure 1.1). Thus, the $n$th pendulum both vibrates near its equilibrium point $x_{n}, i=1,2, \ldots s, s \rightarrow \infty$ and oscillates under force of gravity.

As a dynamic variable we can consider the deviation angle $\phi\left(x_{n}, t\right)$ from the lower vertical position at time $t$, then the potential energy of the elastic interaction between two adjacent pendulums is

$$
\frac{\alpha}{2}\left[\phi\left(x_{n}\right)-\phi\left(x_{n-1}\right)\right]^{2},
$$

where $\alpha$ is the torsion constant.

\footnotetext{
1 For a detailed review of the model and its applications, see, e.g., [63, 85].
} 


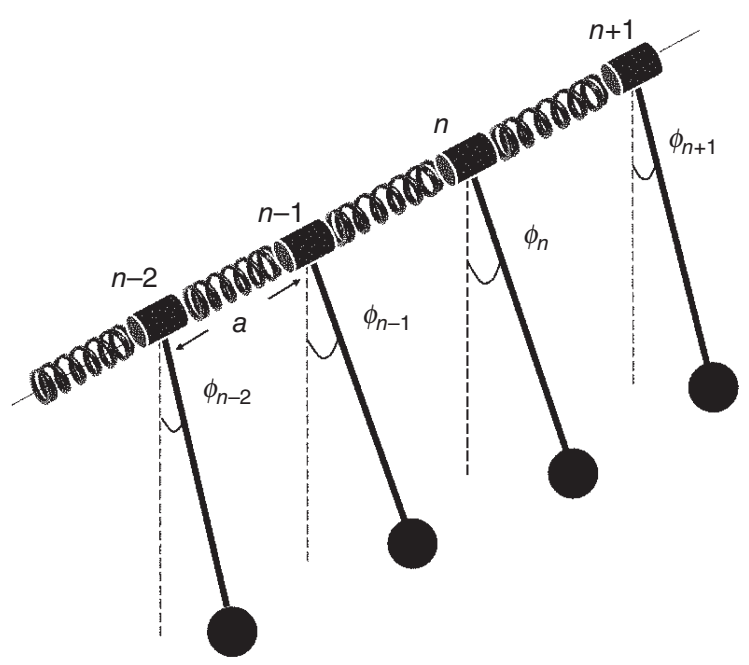

Figure 1.1 Chain of pendulums elastically coupled with their nearest neighbors.

Then the rotational kinetic energy $T$ and the total potential energy $U$ of the system are given by the sum over all the pendulums

$$
\begin{aligned}
T & =\frac{I}{2} \sum_{n}\left(\frac{\partial \phi\left(x_{n}, t\right)}{\partial t}\right)^{2} \\
U & =\frac{\alpha}{2} \sum_{n}\left[\phi\left(x_{n+1}, t\right)-\phi\left(x_{n}, t\right)\right]^{2}+\sum_{n} V\left[x_{n}, t\right],
\end{aligned}
$$

where $I$ is moment of inertia of a pendulum and $x_{n}$ is the coordinate of the $n$th pendulum in the chain. The external potential $V\left[x_{n}\right]$ is simply the gravitational potential energy

$$
V\left[x_{n}\right]=-m g l\left(1-\cos \phi\left(x_{n}, t\right)\right) .
$$

Then the equation of motion of a pendulum placed at $x_{n}$ is

$$
I \frac{\partial^{2} \phi\left(x_{n}, t\right)}{\partial t^{2}}-\alpha\left[\phi\left(x_{n+1}, t\right)-2 \phi\left(x_{n}, t\right)+\phi\left(x_{n-1}, t\right)\right]+m g l \sin \phi\left(x_{n}, t\right)=0 .
$$

Evidently, for small-angle oscillations $\phi\left(x_{n}\right) \ll 1$ this equation can be linearized

$$
I \frac{\partial^{2} \phi\left(x_{n}, t\right)}{\partial t^{2}}-\alpha\left[\phi\left(x_{n+1}, t\right)-2 \phi\left(x_{n}, t\right)+\phi\left(x_{n-1}, t\right)\right]+m g l \phi\left(x_{n}, t\right)=0,
$$

and the motion is simple harmonic. If we neglect the gravity force, the problem is reduced to

$$
I \frac{\partial^{2} \phi\left(x_{n}, t\right)}{\partial t^{2}}-\alpha\left[\phi\left(x_{n+1}, t\right)-2 \phi\left(x_{n}, t\right)+\phi\left(x_{n-1}, t\right)\right]=0 .
$$


This equation can be solved by Fourier transform. Let us multiply (1.4) by $e^{-i k n}$ and sum over all $n$ supposing that $\phi_{n} \equiv \phi\left(x_{n}, t\right)$ decays to zero for large $n$ :

$$
I \frac{\partial^{2}}{\partial t^{2}} \sum_{n=-\infty}^{\infty} \phi_{n} e^{-i k n}=2 \alpha(\cos k-1) \sum_{n=-\infty}^{\infty} \phi_{n} e^{-i k n}
$$

This is the simple differential equation that allows us to find the coefficients of the Fourier transform, the kth mode of the oscillations

$$
\phi(k, t)=\sum_{n=-\infty}^{\infty} \phi_{n} e^{-i k n}
$$

Clearly, the solution is

$$
\phi(k, t)=A(k) \cos (\omega t)+B(k) \sin (\omega t),
$$

where the frequency $\omega=\sqrt{\frac{2 \alpha}{I}(1-\cos k)}$ and $A(k), B(k)$ are arbitrary constants that define the energy of the $k$ th mode. Note that the modes of the linear system are decoupled from each other; there are no transitions between them.

However it is not so easy to find a solution for (1.3) when the linear approximation cannot be used. As a matter of fact one has to apply numerical methods to solve it.

On the other hand, we can consider long-wave excitations in this system. That is, the excitations such that the characteristic length at which $\phi$ changes significantly is much greater than the distance between neighboring pendulums $a$. This allows us to introduce the continuum limit of the model (1.1) replacing the discrete variable $x_{n}$ with the coordinate $x=n a$ and then taking the limit $a \rightarrow 0$.

The Taylor expansion of the functions $\phi\left(x_{n+1}\right)=\phi\left(x_{n}+a\right)$ and $\phi\left(x_{n-1}\right)=$ $\phi\left(x_{n}-a\right)$ yields

$$
\phi\left(x_{n \pm 1}\right) \approx \phi\left(x_{n}\right) \pm a \frac{\partial u\left(x_{n}\right)}{\partial x}+\frac{a^{2}}{2} \frac{\partial^{2} \phi\left(x_{n}\right)}{\partial x^{2}} \pm \frac{a^{3}}{3 !} \frac{\partial^{3} \phi\left(x_{n}\right)}{\partial x^{3}}+\frac{a^{4}}{4 !} \frac{\partial^{4} \phi\left(x_{n}\right)}{\partial x^{4}}+\cdots
$$

Thus, in the order up to $\mathcal{O}\left(a^{4}\right)$,

$$
\phi\left(x_{n+1}\right)-2 \phi\left(x_{n}\right)+\phi\left(x_{n-1}\right) \approx a^{2} \frac{\partial^{2} \phi\left(x_{n}\right)}{\partial x^{2}}
$$

and the equation of motion (1.3) takes the form

$$
I \frac{\partial^{2} \phi(x, t)}{\partial t^{2}}-\alpha a^{2} \frac{\partial^{2} \phi(x, t)}{\partial x^{2}}+m g l \sin \phi(x, t)=0 .
$$

We can now introduce the dimensionless variables to absorb the parameters of the model:

$$
x \rightarrow=\frac{x}{a} \sqrt{\frac{m g l}{\alpha}} ; \quad t \rightarrow t \sqrt{\frac{m g l}{I}} .
$$


Then (1.7) finally takes the form

$$
\frac{\partial^{2} \phi(x, t)}{\partial t^{2}}-\frac{\partial^{2} \phi(x, t)}{\partial x^{2}}+\sin \phi(x, t)=0 .
$$

In other words, in the continuum limit $a \rightarrow 0$ the set of the discrete real angular variables $\phi\left(x_{n}, t\right)$ becomes the scalar field $\phi(x, t)$, which is a continuous canonical variable defined for any coordinate $x$ at any moment of time $t$.

Equation (1.8), known as the sine-Gordon equation, was actually well known long before it got this name. Historically, it was Jacques Edmond Bour [81] who analyzed this equation, considering the compatibility conditions for the Gauss equations for pseudospheres. It was rederived independently by Bonnet in 1867 and Enneper in 1868, again in the context of the differential geometry of surfaces of a constant negative Gaussian curvature. ${ }^{2}$

Consequent study of this equation by Bianchi (1879) and Bäcklund (1880s) [62] resulted in discovery of the interesting result that it is possible to generate a tower of new solutions of (1.8) from one particular known solution, even a trivial one. In Section 1.2 we briefly consider this approach, which is known as the Bäcklund transformation.

Furthermore, (1.8) supports solitonic solutions, the kinks that we discuss in Section 1.2. To the best of our knowledge, these solutions were first found in 1950 in further consideration of the Frenkel-Kontorova model [248], once again a long time before the idea of solitons became fashionable.

In 1962, Perring and Skyrme [319] formulated the sine-Gordon model as a simple, relativistic, nonlinear scalar field theory. Their description is most appropriate for our discussion.

Equation (1.8) may be derived from the Lagrangian

$$
L=\frac{1}{2}\left(\frac{\partial \phi}{\partial t}\right)^{2}-\frac{1}{2}\left(\frac{\partial \phi}{\partial x}\right)^{2}-U[\phi] \equiv \frac{1}{2} \partial_{\mu} \phi \partial^{\mu} \phi-U(\phi),
$$

where for the sake of generality we introduce the potential of the scalar field $U(\phi)=(1-\cos \phi)$. Hereafter we make use of the covariant notations in $1+1$ dimensions to make manifest the Lorenz invariance of the model. Our choice for the metric in $1+1$ dimensions is $g_{\mu \nu}=\operatorname{diag}(1,-1)$ and we adopt the natural units $\hbar=c=1$ to simplify our notations. The corresponding variational equation is

$$
\frac{\partial}{\partial x_{\mu}}\left(\frac{\delta L}{\delta\left(\partial_{\mu} \phi\right)}\right)=\frac{\delta L}{\delta \phi},
$$

which yields the covariant form of (1.8)

$$
\partial_{\mu} \partial^{\mu} \phi=-U^{\prime}(\phi)
$$

2 There were (unsuccessful) attempts to restore historical credit, e.g., referring to this equation to as the Enneper (sine-Gordon) equation [358]. 
Evidently, the canonical stress energy tensor is

$$
T_{\mu \nu}=\left(\frac{\delta L}{\delta\left(\partial^{\mu} \phi\right)}\right) \partial_{\nu} \phi-g_{\mu \nu} L=\partial_{\mu} \phi \partial_{\nu} \phi-g_{\mu \nu} L
$$

Explicitly, the components of $T_{\mu \nu}$ are

$$
\begin{array}{ll}
T_{00}=\frac{1}{2}\left(\frac{\partial \phi}{\partial t}\right)^{2}+\frac{1}{2}\left(\frac{\partial \phi}{\partial x}\right)^{2}+1-\cos \phi ; & T_{01}=\frac{\partial^{2} \phi}{\partial x \partial t} \\
T_{11}=\frac{1}{2}\left(\frac{\partial \phi}{\partial t}\right)^{2}+\frac{1}{2}\left(\frac{\partial \phi}{\partial x}\right)^{2}-1+\cos \phi ; & T_{10}=\frac{\partial^{2} \phi}{\partial x \partial t}
\end{array}
$$

As usual, this tensor is conserved due to translational symmetry of the sineGordon model, i.e.,

$$
\partial_{\mu} T^{\mu \nu}=\left\{\begin{array}{l}
\partial_{t} T^{00}-\partial_{x} T^{10}=0 \\
\partial_{t} T^{01}-\partial_{x} T^{11}=0 .
\end{array}\right.
$$

Since we are interested in finite-energy solutions, we have to consider the total energy of this system

$$
E=\int_{-\infty}^{\infty} d x T_{00}=\int_{-\infty}^{\infty} d x\left[\frac{1}{2}\left(\partial_{t} \phi\right)^{2}+\frac{1}{2}\left(\partial_{x} \phi\right)^{2}+U(\phi)\right] .
$$

The corresponding vacuum solutions of the field equation (1.11) are configurations $\phi_{0}$, which satisfy the stationary points of the action, i.e., we shall search for fields that satisfy the conditions

$$
U^{\prime}\left(\phi_{0}\right)=0 ; \quad U^{\prime \prime}\left(\phi_{0}\right)>0
$$

The potential of the sine-Gordon model $U(\phi)=(1-\cos \phi)$ is periodic. It has an infinite number of degenerate vacua at $\phi_{0}=2 \pi n, \quad n \in \mathbb{Z}$, for each of those $U^{\prime \prime}\left(\phi_{0}\right)=1$.

Lorentz invariance of the model (1.9) allows us to start from the static configurations; they can be boosted if necessary. Therefore, we suppose that $\partial_{0} \phi=0$ and the energy functional (1.15) can be written as

$$
E=\int_{-\infty}^{\infty} d x\left[\frac{1}{\sqrt{2}} \partial_{x} \phi \pm \sqrt{U(\phi)}\right]^{2} \mp \int_{-\infty}^{\infty} d x \sqrt{2 U(\phi)} \partial_{x} \phi \geqslant 0
$$

Evidently, the energy is minimal if

$$
\frac{1}{2}\left(\frac{\partial \phi}{\partial x}\right)^{2}=U(\phi)
$$


We suppose that the potential is positively defined for any values of $\phi(x)$, thus we can define a superpotential $W(\phi)$ as a function associated with $U(\phi)$ :

$$
\frac{1}{2}\left(\frac{\partial W}{\partial \phi}\right)^{2}=U(\phi)
$$

Hence, the second term in (1.17) can be written as

$$
\int_{-\infty}^{\infty} d x \sqrt{2 U(\phi)} \partial_{x} \phi=W[\phi(\infty)]-W[\phi(-\infty)] .
$$

Then the lower energy bound (so-called Bogomolny bound [77]) is saturated if $E \geqslant W[\phi(\infty)]-W[\phi(-\infty)]$ and the scalar field satisfies the first-order equation

$$
\frac{\partial \phi}{\partial x}= \pm \frac{\partial W}{\partial \phi}
$$

The idea of superpotential $W(\phi)$ actually originates from supersymmetric models where this function becomes a fundamental quantity (see, e.g., [354]). On the other hand, the method of superpotential is very useful to construct nontrivial soliton solutions in a system of coupled scalar fields [60].

A trivial vacuum solution of the field equation (1.8) is simply the state $\phi=$ $\phi_{0}=$ const, where $\phi_{0}$ are the minima of the potential energy $U(\phi)$. Then the Bogomolny bound is saturated trivially, i.e., $W[\phi(\infty)]=W[\phi(-\infty)]$ and $E \geqslant 0$.

We can consider small oscillations about the vacuum, then the Taylor expansion yields $U\left(\phi-\phi_{0}\right) \approx \frac{1}{2}\left(\phi-\phi_{0}\right)^{2}$ and we arrive at the original linear KleinGordon equation for the scalar field of unit mass:

$$
\left(\partial_{t}^{2}-\partial_{x}^{2}+1\right) \phi=0
$$

A plane-wave solution of this equation, commonly referred to as a mode, is

$$
\phi_{k, \omega}(x, t)=A e^{i(k x-\omega t)},
$$

where $A$ is the amplitude, $k$ is the wavenumber of the mode and $\omega$ is the frequency of the propagating wave. Substitution of this function into (1.22) yields the dispersion relation

$$
-\omega^{2}+k^{2}+1=0 .
$$

In other words, the linear waves of different lengths propagate with different speeds. Thus, a general solution of the Klein-Gordon equation can be written as an integral sum over all modes

$$
\phi(x, t)=\int_{-\infty}^{\infty} d x\left\{A_{+}(k) e^{i\left(k x-\omega_{+} t\right)}+A_{-}(k) e^{i\left(k x-\omega_{-} t\right)}\right\},
$$


where $\omega_{ \pm}= \pm \sqrt{k^{2}+1}$. Evidently, this is a usual expansion in a Fourier series. These states belong to the perturbative sector of the model, in the context of the discrete Frenkel-Kontorova model (1.1) these linear excitations can be identified with phonons.

The solution of the sine-Gordon equation is trivial if the field is in the vacuum state, i.e., $\phi_{0}=2 \pi n, \quad n \in \mathbb{Z}$. Since we are looking for a regular solution with finite total energy, the field must approach the vacuum as $x \pm \infty$ and $\partial_{x} \phi(x, t) \rightarrow 0$ as $x \rightarrow \pm \infty$. We also suppose that $\partial_{t} \phi(x, t)$ is bounded on both ends of the infinite one-dimensional space.

However, the vacuum is infinitely degenerated and the corresponding vacua can be different. For example, we can consider the asymptotic conditions $\phi(-\infty)=0$ and $\phi(\infty)=2 \pi$. Then the field is not in the vacuum everywhere; it is interpolating between these two vacuum values and the corresponding potential energy of the configuration is no longer zero. Note that in that case the transition to the trivial solution is not possible-the boundary conditions on the field are different for these configurations and it would take an infinite amount of energy to overcome the barrier between these two sectors. ${ }^{3}$

To find nontrivial solutions of the sine-Gordon equation (1.8) let us consider the first-order equation (1.18). Evidently, for the case under consideration $U(\phi)=(1-\cos \phi)$ and the superpotential is $W(\phi)=-4 \cos \frac{\phi}{2}$. Thus, the minimal energy bound is saturated if

$$
\frac{\partial \phi}{\partial x}= \pm 2 \sin \frac{\phi}{2} .
$$

Separating the variables, we arrive to

$$
d x= \pm \frac{d(\phi / 2)}{\sin (\phi / 2)}
$$

Let us consider the positive sign in the right-hand side of this equation. Then the integration yields

$$
x-x_{0}=\ln \tan \frac{\phi}{4},
$$

where $x_{0}$ is the integration constant. Thus, we get the nontrivial solution to the sine-Gordon model

$$
\phi_{K}(x)=4 \arctan e^{x-x_{0}} .
$$

This solution is referred to as the kink. It corresponds to the transition between two neighboring vacua, as at $x \rightarrow-\infty$ the field is taking the value $\phi(-\infty)=0$ while at $x \rightarrow \infty$ it approaches another vacuum value, $\phi(\infty)=2 \pi$ (see Figure 1.1).

3 Strictly speaking, this energy is proportional to the volume of the 1-dim space L. 

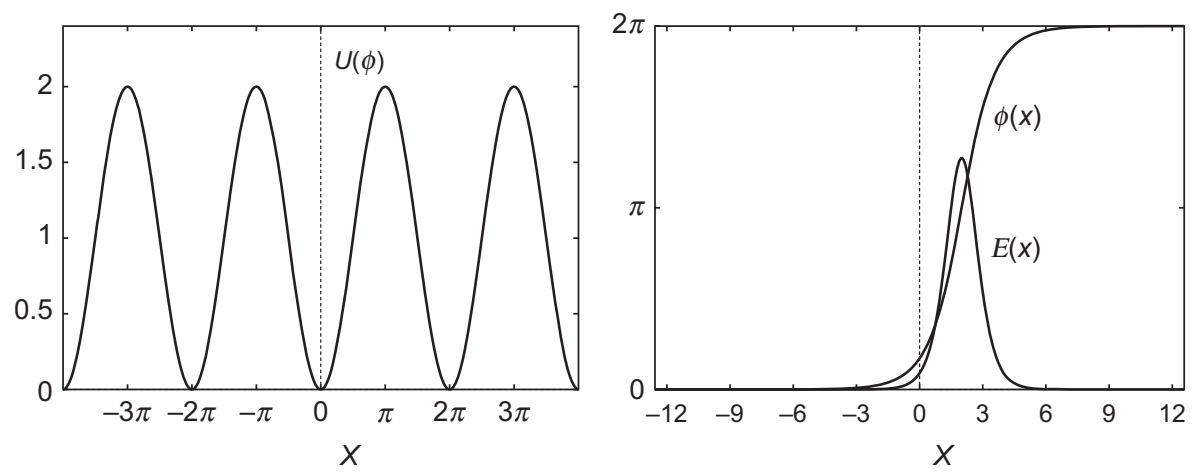

Figure 1.2 The potential of the sine-Gordon model (left panel), the energy density of the static kink, and the kink solution localized at $x_{0}=2$ (right panel).

Obviously, taking the negative sign in the right-hand side of (1.27) we obtain another solution, which interpolates between $\phi(-\infty)=2 \pi$ and $\phi(\infty)=0$, the antikink

$$
\phi_{\bar{K}}(x)=4 \arctan e^{-\left(x-x_{0}\right)} .
$$

Both kink and antikink are exponentially localized lumps of energy centered around the $x=x_{0}$. Indeed, substitution of the static solutions (1.28) or (1.29) into the integrand of (1.15) yields the energy-density distribution displayed in Figure 1.2, right panel.

$$
E(x)=\frac{4}{\cosh ^{2}\left(x-x_{0}\right)} .
$$

The dimensionless energy of the static configuration, i.e., its mass, is finite,

$$
M=\int_{-\infty}^{\infty} d x E(x)=8 .
$$

This solution is an example of a soliton, a spacially localized particle-like configuration that is stable and, in many respects, behaves like a particle. The kink state belongs to the non-perturbative sector of the sine-Gordon model; it cannot be obtained via perturbative expansion in the vicinity of a particular vacuum since it becomes infinitely heavy in the weak-coupling limit. Furthermore, the kink solution is a topological soliton. We can introduce the topological current

$$
j_{\mu}=\frac{1}{2 \pi} \varepsilon_{\mu \nu} \partial^{\nu} \phi ; \quad \partial^{\mu} j_{\mu}=\frac{1}{2 \pi} \varepsilon_{\mu \nu} \partial^{\mu} \partial^{\nu} \phi \equiv 0,
$$


which is automatically conserved. Notably, it is not a Noether current, associated with some symmetry of the model. ${ }^{4}$ Thus, the topological charge is

$$
Q=\frac{1}{2 \pi} \int_{-\infty}^{\infty} d x \frac{\partial \phi}{\partial x}=\frac{1}{2 \pi}[\phi(\infty)-\phi(-\infty)]
$$

The topological charge is an index that labels different sectors of the sine-Gordon model. The kink configuration (1.28) corresponds to the sector with $Q=1$, while the antikink solution (1.29) belongs to the sector with $Q=-1$. The trivial vacuum solution is in the sector with $Q=0$. Note that in the framework of the Frenkel-Kontorova model the kink solitons are representing dislocations in a onedimensional atomic chain. These solutions are important in solid-state physics to model various plastic deformations.

In what follows we use the properties of symmetry of the sine-Gordon equation (1.8). The model is symmetric both with respect to the usual space-time symmetries, i.e., translations $x \rightarrow x+x_{0}, t \rightarrow t+t_{0}$, reflections $x \rightarrow-x, t \rightarrow-t$, and the Lorentz transformations (recall that we are using natural units)

$$
x \rightarrow \frac{x-v t}{\sqrt{1-v^{2}}}=\gamma(x-v t) ; \quad t \rightarrow \frac{t-v x}{\sqrt{1-v^{2}}}=\gamma(t-v x),
$$

where $\gamma=1 / \sqrt{1-v^{2}}$ is the Lorentz factor, as usual.

The sine-Gordon model also enjoys another group of symmetries, which include the reflections of the field $\phi \rightarrow-\phi$ and shifts between the vacua $\phi \rightarrow \phi+2 \pi n$. Thus, the moving solitons may be obtained by a Lorentz transformation of the kink solution (1.28):

$$
\phi_{K}(x, t)=4 \arctan \exp \left(\gamma\left(x+x_{0}+v t\right)\right) .
$$

Then we can also define the momentum of the moving configuration as

$$
P=\int_{-\infty}^{\infty} d x T_{1}^{0}=\int_{-\infty}^{\infty} d x \partial_{x} \phi \partial_{t} \phi
$$

Clearly, both the energy of the kink and its momentum conserve (cf. (1.14)). Substitution of the solution (1.34) into the expression (1.35) yields the explicit value of the momentum $P=8 v / \sqrt{1-v^{2}}$.

4 However, in the quantum-field theory there is an exact equivalence between the sine-Gordon model and the massive Thirring model [105]. This duality allows us to identify the topological current of the quantum sine-Gordon model with dual $U(1)$ Noether current of the massive Thirring model. 


\subsection{Bäcklund Transformation and Other Solutions for the Sine-Gordon Equation}

The sine-Gordon equation (1.8) is a nonlinear hyperbolic partial differential equation, thus unlike the Klein-Gordon equation (1.22), a linear combination of two solutions of the former equation is not a solution. However, the most remarkable property of the sine-Gordon equation (1.8) is related to its symmetries. There is a nice and very rare possibility to construct all solutions of it, both in perturbative and non-perturbative sectors.

Before proceeding with discussion of the solutions of the sine-Gordon equation, let us make a few mathematical comments. Historically, the sine-Gordon equation arose from investigation of the geometry of isometries of surfaces with constant negative Gaussian curvature contained in $\mathbb{R}^{3}[62]$. Now, we can introduce the light-cone coordinates

$$
\tau=\frac{1}{2}(x+t) ; \quad \rho=\frac{1}{2}(x-t),
$$

thus

$$
\partial_{x}=\frac{1}{2}\left(\partial_{\tau}+\partial_{\rho}\right) ; \quad \partial_{t}=\frac{1}{2}\left(\partial_{\tau}-\partial_{\rho}\right) .
$$

Then the usual d'Alembert operator in $1+1$ dimensions can be written as $\square=\partial_{t}^{2}-\partial_{x}^{2}=-\partial_{\tau} \partial_{\rho}$. In the light-cone coordinates (1.36), the sine-Gordon equation (1.8) becomes simple

$$
\phi_{\rho \tau}=\sin \phi
$$

Note that the usual linear wave equation in this variables becomes $\phi_{\rho \tau}=0$.

On the other hand, we can consider a two-dimensional surface covered by these coordinates (known as Tchebyshev nets; see, e.g., [335]) with the line element (first fundamental form of the surface)

$$
d s^{2}=d \tau^{2}+d \rho^{2}+2 \cos \phi d \tau d \rho,
$$

where $\phi$ is the angle between the asymptotic lines $\tau=$ const, $\rho=$ const on the surface embedded into Euclidean space $\mathbb{R}^{3}$, which can be taken as parametric curves. ${ }^{5}$ In other words, the coordinates $\rho, \theta$ correspond to the parametrization of the surface by arc length along asymptotic lines. Then the corresponding second fundamental form $2 \sin \phi d \tau d \rho$ is zero along the asymptotic directions and the Gaussian curvature $K$ satisfies the equation $\partial_{\tau} \partial_{\rho} \phi+K \sin \phi=0$. Thus, the sineGordon equation (1.38) in the light-cone coordinates (1.36), is the compatibility equation between the first and second fundamental forms of a surface; it implies that the Gaussian curvature of this pseudospherical surface is constant: $K=-1$.

5 In the original $x, t$ coordinates this form can be written as

$$
d s^{2}=\sin ^{2} \frac{\phi}{2} d t^{2}+\cos ^{2} \frac{\phi}{2} d x^{2} .
$$




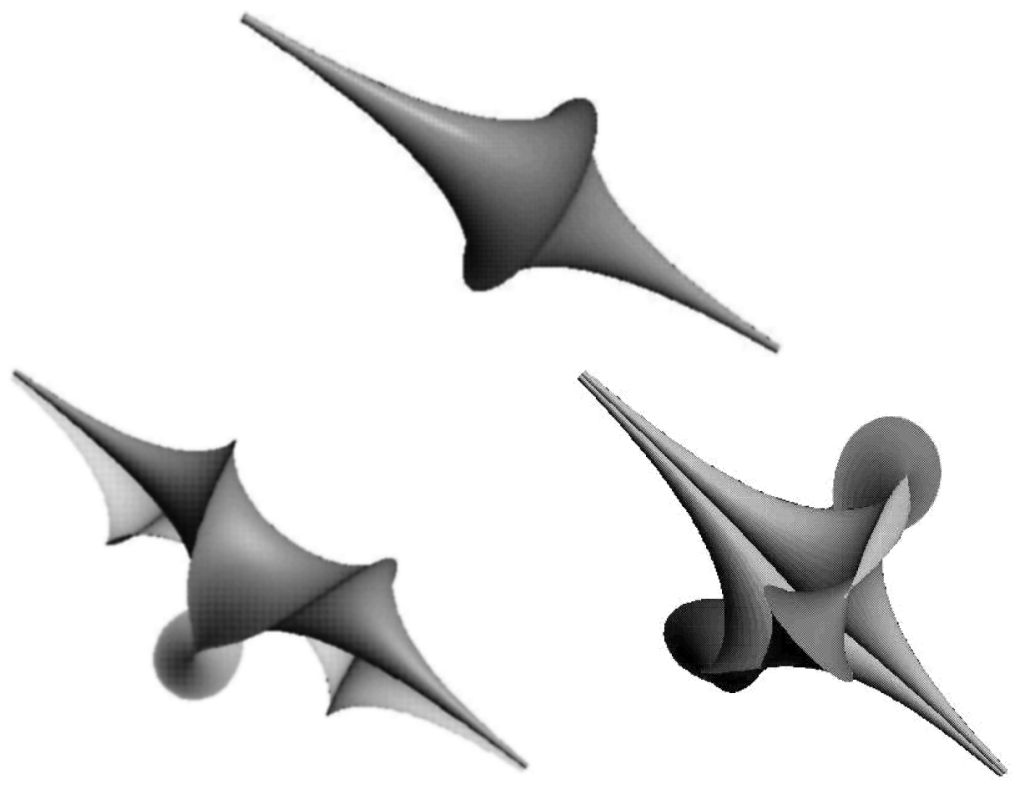

Figure 1.3 Pseudospherical surfaces of Gaussian curvature $K=-1$, which correspond to the kink solution (1.28) (upper plot), two-kink solution (1.49) (bottom left), and breather solution (1.62) (bottom right). (Plots were generated with the visualization program 3D-XplorMath).

The surface that corresponds to the kink configuration (1.28) is displayed in Figure 1.3, upper plot.

The form (1.38) of the sine-Gordon equation is convenient to construct a variety of solutions. Geometrically, these solutions can be obtained via a simple geometrical transformation of pseudospherical surfaces with the same curvature (see, e.g., [335]). Analytically, the statement is that one can construct a new solution of the sine-Gordon equation, starting from a given solution, even the trivial one, $\phi=0$. This gives us an algorithm of generation of an infinite tower of solitons for the sine-Gordon hierarchy. More generally, this nice possibility is related to a special feature of (1.8), the sine-Gordon system is completely integrable.

Roughly speaking, a classical Hamiltonian system is referred to as integrable if it possesses infinitely many integrals of motion. In Section 1.3, we briefly comment on this beautiful property; here we just note that the presence of the soliton solutions in the spectrum is not directly related to integrability of the model, though there many integrable systems that support various solitons.

The Bäcklund transformation for the sine-Gordon equation starts from the observation that the second-order equation (1.8) can be represented in terms of two first-order equations. Suppose that the function $\phi_{0}(\tau, \rho)$ is a particular solution of the sine-Gordon equation, i.e., $\partial_{\tau} \partial_{\rho} \phi_{0}=\sin \phi_{0}$. 
Let us now consider another function $\phi_{1}(\tau, \rho)$ that satisfies the system of two equations

$$
\begin{aligned}
& \partial_{\tau} \phi_{1}=\partial_{\tau} \phi_{0}-2 \lambda \sin \left(\frac{\phi_{0}+\phi_{1}}{2}\right), \\
& \partial_{\rho} \phi_{1}=-\partial_{\rho} \phi_{0}+\frac{2}{\lambda} \sin \left(\frac{\phi_{0}-\phi_{1}}{2}\right),
\end{aligned}
$$

where $\lambda$ is a parameter of the transformation.

Suppose there is the compatibility condition, which can be obtained by crossdifferentiating the function $\phi_{1}$, and the partial derivatives are commuting, i.e.,

$$
\partial_{\rho} \partial_{\tau} \phi_{1}=\partial_{\tau} \partial_{\rho} \phi_{1}
$$

Then, differentiating the first equation with respect to $\rho$ and the second equation with respect to $\tau$, and making use of the original equations (1.39), we obtain

$$
\begin{aligned}
\partial_{\rho} \partial_{\tau} \phi_{1} & =\partial_{\rho} \partial_{\tau} \phi_{0}-2 \cos \left(\frac{\phi_{0}+\phi_{1}}{2}\right) \sin \left(\frac{\phi_{0}-\phi_{1}}{2}\right) \\
& =\partial_{\rho} \partial_{\tau} \phi_{0}+\sin \phi_{0}-\sin \phi_{1} .
\end{aligned}
$$

Hence, if $\phi_{0}$ satisfies the sine-Gordon equation, the function $\phi_{1}$ also is a solution, though not necessarily the same.

Indeed, let us begin from the trivial solution $\phi_{0}=0$. Then the system of equations (1.39) is reduced to

$$
\partial_{\tau} \phi_{1}=-2 \lambda \sin \left(\phi_{1} / 2\right) ; \quad \partial_{\rho} \phi_{1}=-2 \lambda^{-1} \sin \left(\phi_{1} / 2\right) .
$$

Now, separating the variables, we get another solution, which can be easily identified as the one-kink soliton (1.34):

$$
\phi_{1}(\tau, \rho)=4 \arctan \exp \left(-\lambda \tau-\frac{\rho}{\lambda}+\delta\right),
$$

where $\delta$ is the integration constant.

Now we can fix the value of the parameters $\lambda, \delta$. Indeed, recovering the original variables $x, t$ by comparing this solution with (1.34) we can see that $\delta \longmapsto x_{0}$ and

$$
-\lambda \tau-\frac{\rho}{\lambda}=-\frac{1}{2}\left(\lambda+\frac{1}{\lambda}\right) x-\frac{1}{2}\left(\lambda-\frac{1}{\lambda}\right) t \equiv \frac{x+v t}{\sqrt{1-v^{2}}} .
$$

Thus,

$$
v=\frac{1-\lambda^{2}}{1+\lambda^{2}} \quad \text { or } \quad \lambda= \pm \sqrt{\frac{1-v}{1+v}} .
$$

Note that we generated both the kink and antikink solutions.

It is convenient to write the one-kink solution in the form

$$
\phi_{1}=4 \arctan e^{\theta}, \quad \theta=-\left[\frac{\lambda^{2}+1}{2 \lambda}\left(x+\frac{\lambda^{2}-1}{\lambda^{2}+1} t\right)+\delta\right] .
$$


We can now continue this process, i.e., substitute the new solution $\phi_{1}(\tau, \rho)$ (1.42) into the system of the Bäcklund equations (1.39) and integrate it to get another soliton solution (presumably this procedure will give us a two-kink solution), etc. However, at that step the straightforward integration becomes rather involved, so there is another way around.

Indeed, a remarkable property of the Bäklund transformation (1.39) is that the successive transformations with different choices of parameters $\lambda_{1}$ and $\lambda_{2}$ commute. ${ }^{6}$ In other words, performing two consequent transformations

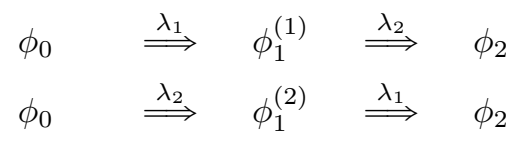

as a first step we obtain two different one-kink solitons (1.42), and then we get a new solution composing these solitons into the KK system of charge $Q=2$ as a second step. Thus, we have four pairs of equations:

$$
\begin{array}{lll}
\partial_{\tau} \phi_{0}-\partial_{\tau} \phi_{1}^{(1)}=2 \lambda_{1} \sin \frac{\phi_{0}+\phi_{1}^{(1)}}{2}, & \partial_{\rho} \phi_{0}+\partial_{\rho} \phi_{1}^{(1)}=\frac{2}{\lambda_{1}} \sin \frac{\phi_{0}-\phi_{1}^{(1)}}{2} \\
\partial_{\tau} \phi_{0}-\partial_{\tau} \phi_{1}^{(2)}=2 \lambda_{2} \sin \frac{\phi_{0}+\phi_{1}^{(2)}}{2}, & \partial_{\rho} \phi_{0}-\partial_{\rho} \phi_{1}^{(2)}=\frac{2}{\lambda_{2}} \sin \frac{\phi_{0}-\phi_{1}^{(2)}}{2} \\
\partial_{\tau} \phi_{2}-\partial_{\tau} \phi_{1}^{(1)}=-2 \lambda_{2} \sin \frac{\phi_{1}^{(1)}+\phi_{2}}{2}, & \partial_{\rho} \phi_{2}+\partial_{\rho} \phi_{1}^{(1)}=\frac{2}{\lambda_{2}} \sin \frac{\phi_{1}^{(1)}-\phi_{2}}{2} \\
\partial_{\tau} \phi_{2}-\partial_{\tau} \phi_{1}^{(2)}=-2 \lambda_{1} \sin \frac{\phi_{1}^{(2)}+\phi_{2}}{2}, & \partial_{\rho} \phi_{2}+\partial_{\rho} \phi_{1}^{(2)}=\frac{2}{\lambda_{1}} \sin \frac{\phi_{1}^{(2)}-\phi_{2}}{2} .
\end{array}
$$

Simple algebraic transformations allow us to eliminate the derivatives, thus in both cases we obtain the same relation

$$
\lambda_{1} \sin \frac{1}{4}\left(\phi_{0}+\phi_{1}^{(2)}-\phi_{1}^{(1)}-\phi_{2}\right)=\lambda_{2} \sin \frac{1}{4}\left(\phi_{0}+\phi_{1}^{(1)}-\phi_{1}^{(2)}-\phi_{2}\right) .
$$

This finally yields the addition formula for the algebraic Bäcklund transformation:

$$
\tan \frac{\phi_{2}-\phi_{0}}{4}=\frac{\lambda_{1}+\lambda_{2}}{\lambda_{1}-\lambda_{2}} \tan \frac{\phi_{1}^{(2)}-\phi_{1}^{(1)}}{4} .
$$

Consequently, we can apply this algebraic relation to generate recursively new solutions of the sine-Gordon model of higher degree, step by step. Note that at each step the number of parameters of the solution increases. The one-kink solution depends on two parameters, $\lambda$ and $\delta$, and for the two-kink configuration we have four free parameters, $\lambda_{1}, \lambda_{2}$ and $x_{0}^{(1)}, x_{0}^{(2)}$, etc. Physically, taking different values of those parameters we can specify positions, topological charges of the constituents, and velocities.

6 More precisely, they commute for a special choice of the integration constants. 

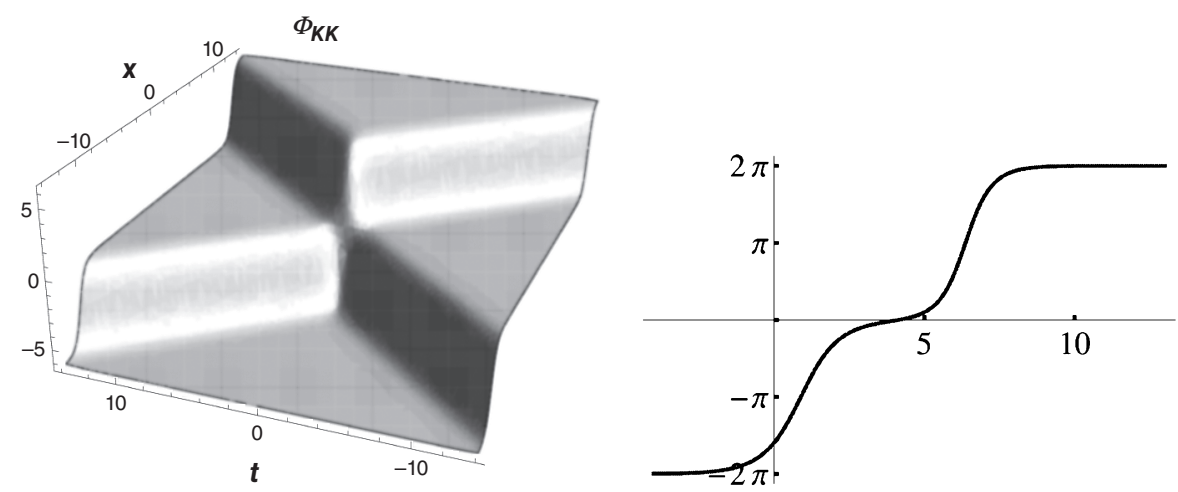

Figure 1.4 Dynamics of the symmetric head-on KK collision for $v=0.8$ (1.47) and the field of the symmetric two-kink configuration at $\lambda_{1}=-\lambda_{2}=0.6$ and $\delta_{1}=\delta_{2}=0$.

A particular example is the symmetric two-kink solution centered at the origin. Let us take $\lambda_{2}=-1 / \lambda_{1}, x_{0}^{(1)}=x_{0}^{(2)}=0$, i.e., $v_{1}=v_{2}=v$, and for the sake of definiteness consider positive values of $\lambda_{1}$. Then $\phi_{0}=0$ and the addition formula (1.46) yields

$$
\phi_{2}=\phi_{K K}=4 \tan ^{-1}\left(\frac{v \sinh \gamma x}{\cosh \gamma v t}\right),
$$

where $v=\left(1-\lambda_{1}^{2}\right) /\left(1+\lambda_{1}^{2}\right)$. One can easily verify that the topological charge of this configuration is

$$
Q=\frac{1}{2 \pi}\left[\phi_{2}(\infty)-\phi_{2}(-\infty)\right]=2,
$$

as expected. Indeed, on the spacial infinity $\phi_{K K}( \pm \infty, t)= \pm 2 \pi$ and $\phi_{2}(0, t)=0$, thus the field varies from $-2 \pi$ to 0 along the negative half line, and from 0 to $2 \pi$ along positive half line (see Figure 1.4, right panel).

Note that this configuration has constituent structure; it is not a function of a single variable $x-v t$. The $\phi_{K K}$ system (1.49) consists of two kinks moving with different velocities, however the solitons became individual only when they are well separated. The constituent structure of the configuration (1.47) becomes evident when we evaluate the corresponding energy and momentum of the field. Indeed, substitution of the two-kink solution (1.47) into the definitions of the energy (1.15) and momentum (1.35), gives

$$
E=\frac{8}{\sqrt{1-v_{1}^{2}}}+\frac{8}{\sqrt{1-v_{2}^{2}}} ; \quad P=\frac{8 v_{1}}{\sqrt{1-v_{1}^{2}}}-\frac{8 v_{2}}{\sqrt{1-v_{2}^{2}}} .
$$

Since the mass of the static kink in dimensionless variables is $M_{0}=8$, the contribution of each component remains clearly individual for any moment of time. Note that the solutions we obtain in such a way are not static; the 
configuration (1.47) is ill defined in the limit $v=0$ and the constituents are always doomed to move. In Figure 1.4 we display the entire process of the collision of two kinks in the axis $x, t$.

Another particular example is the symmetric kink-antikink collision. Considering $\lambda_{2}=1 / \lambda_{1}, x_{0}^{(1)}=x_{0}^{(2)}=0$ we get $v_{1}=-v_{2}=v$, i.e., the soltons are moving with equal and opposite velocity. In this case

$$
\phi_{2}=\phi_{K \bar{K}}=4 \tan ^{-1}\left(\frac{\sinh \gamma v t}{v \cosh \gamma x}\right) \text {, }
$$

or

$$
\phi_{K \bar{K}}=4 \tan ^{-1}\left[\frac{e^{\gamma v t-\ln v}-e^{-\gamma v t-\ln v}}{e^{\gamma x}+e^{-\gamma x}}\right] .
$$

The soliton solutions of the sine-Gordon model are bona fide solitons; the asymptotical structure of the two-kink configuration in the process of the collision is different by the phase shift only. To find its value we have to compare the spacial asymptotic of the initial configuration (1.48) at $t=-\infty$ with the final asymptotic of infinitely separated scattered kinks at $t=\infty$.

Let us consider the solution (1.48) in the limit of very large negative time $t \rightarrow-\infty$, then expansion of the exponents yields the asymptotic form of the initial configuration

$$
\phi_{K \bar{K}} \approx \phi_{K}\left[x+v\left(t+\frac{\delta t}{2}\right) \gamma\right]+\phi_{\bar{K}}\left[x-v\left(t-\frac{\delta t}{2}\right) \gamma\right],
$$

where $\phi_{K}$ and $\phi_{\bar{K}}$ are the kink and antikink solutions (1.34), respectively. The parameter $\delta t=2 \frac{\ln v}{\gamma v}$ defines the shift of the position of scattered solitons; it is actually a delay of the time experienced by a kink due to interaction with an antikink. Indeed, in the remote future $t \rightarrow \infty$ the solution (1.48) becomes

$$
\phi_{K \bar{K}} \approx \phi_{K}\left[x+v\left(t-\frac{\delta t}{2}\right) \gamma\right]+\phi_{\bar{K}}\left[x-v\left(t+\frac{\delta t}{2}\right) \gamma\right] .
$$

In other words, the scattering of the kinks in the sine-Gordon model is dispersionless.

To construct a general two-kinks solution of the sine-Gordon equation we have to consider the one-kink solitons written in the form (1.44) with arbitrary values of the parameters, i.e., $\phi_{i}=4 \tan ^{-1} e^{\theta_{i}}, i=1,2$. Then we can apply the addition formula (1.46). The result of some algebraic manipulations is the two-soliton solution

$$
\phi_{2}=4 \tan ^{-1}\left(\frac{\lambda_{1}+\lambda_{2}}{\lambda_{1}-\lambda_{2}}\right) \frac{e^{\theta_{1}}-e^{\theta_{2}}}{1+e^{\theta_{1}+\theta_{2}}} .
$$

This formula actually contains information about the process of collision of the kinks. The initial configuration $\phi_{2}$ at $t=-\infty$ consists of two infinitely separated kinks that collide at $t=0$ and then become separated again. Clearly, 

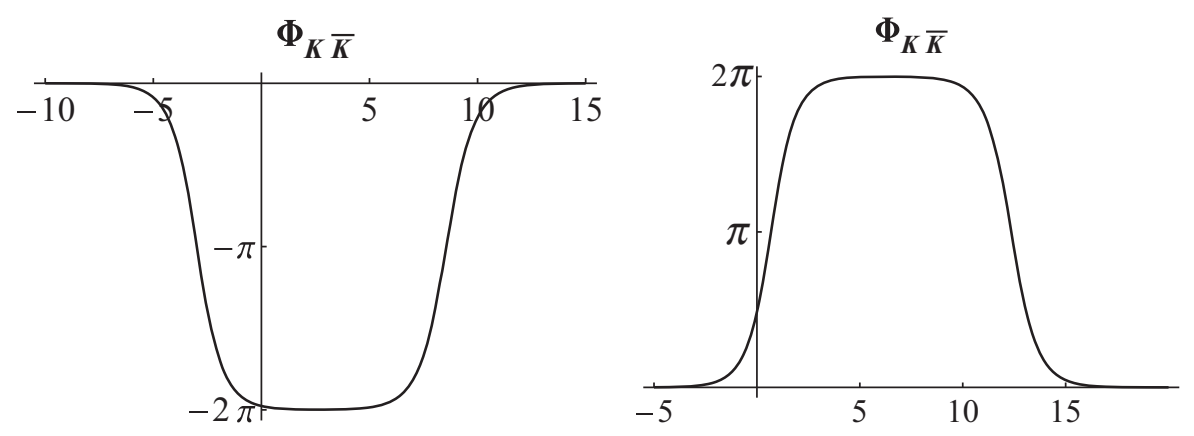

Figure 1.5 The field of the kink-antikink configuration (1.48) at $\lambda_{1}=\lambda_{2}=0.4$ and $\delta_{1}=-\delta_{2}=5$ before and after the head-on $K \bar{K}$ collision for $v=0.6$.

all other degrees of freedom of the model do not contribute to this process and production of the kink-antikink pairs in the sine-Gordon model is not allowed. The remarkable point is that the radiation modes are not excited by the collision of the kinks; they do not appear in the final state after the collision of the solitons. Thus, the perturbative and non-perturbative sectors of the sine-Gordon model are completely isolated from each other. Here we can see another manifestation of the integrability of the model; it is completely solvable and all solutions can be presented analytically in closed form.

One would naively expect that the collision of the kink and antikink at low impact velocity would mean their annihilation into the trivial vacuum. However, the integrability of the sine-Gordon model does not allow this to happen. Indeed, though the configuration (1.48) belongs to the topologically trivial sector, the collision between the kink and antikink is completely elastic. The final state after collision in the remote future describes an infinitely separated kink and antikink. The only difference from the central symmetric collision of the kinks is the flip of the vacuum. While in the initial state the kink and antikink were interpolating between the neighboring vacua 0 on both ends of the spacial infinite line and $-2 \pi$ in the center of collision, in the final state they approach the vacuum $2 \pi$ at the center and $\phi(x \rightarrow \pm \infty)=0$. This scenario is displayed in Figure 1.5. Similar to the collision of the kinks, the positions of the solitons are shifted by $2 \delta$.

\subsubsection{Excitations of the Kink and Interaction between the Solitons}

It would be misleading to conclude that the kinks do not interact with each other; there is always a force acting between the solitons. This force can be evaluated when we consider the limit of the expression (1.47) with the kinks separated by a finite large distance $d$. Then the asymptotic interaction energy can be found if we consider an initial configuration

$$
\phi(x)=\phi_{K}(x+d)+\phi_{K}(x-d)-2 \pi,
$$




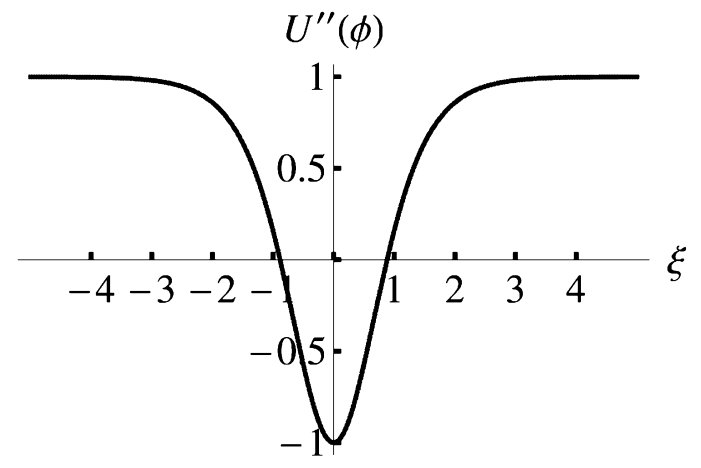

Figure 1.6 The modified Pöschl-Teller potential of the linear excitations of the sine-Gordon model.

where $\phi_{K}(x)$ is the one-kink solution (1.28). Then we can expand the corresponding energy of the configuration in powers of $1 / d$ and subtract the mass of two infinitely separated kinks, $E_{\text {int }}(d)=E_{K K}(d)-2 M_{0}$, where $M_{0}=8$. This yields the interaction energy [319]

$$
E_{\text {int }}=32 e^{-2 d}
$$

Evidently, it is the Yukawa-type interaction, and it is repulsive for the kinks and attractive in the case of the kink-antikink pair.

Indeed, let us consider small excitations $\eta(x, t)$ about the sine-Gordon kink $(1.28), \phi_{K}(x)+\eta(x, t)$, where $\phi_{K}(x)=4 \arctan \left(e^{x}\right)$. Then, the equation that describes these deviations from the kink profile can be easily derived from (1.8):

$$
\frac{\partial^{2} \eta(x, t)}{\partial t^{2}}-\frac{\partial^{2} \eta(x, t)}{\partial x^{2}}+\eta(x, t) \cos \phi_{K}(x)=0 .
$$

Now we can separate the variables: $\eta(x, t)=\xi(x) e^{i \omega t}$. After some simple algebra we arrive at the linear, one-dimensional, Schrödinger-type equation on the fluctuation eigenfunctions

$$
\left(-\frac{d^{2}}{d x^{2}}+1-\frac{2}{\cosh ^{2} x}\right) \xi(x)=\omega^{2} \xi(x) .
$$

This equation is very well known. The corresponding Pöschl-Teller potential (Figure 1.6) was proposed in 1933 to model diatomic molecules [326] ${ }^{7}$

$$
\left.\frac{\partial^{2} U(\phi)}{\partial \phi^{2}}\right|_{\phi=\phi_{K}}=1-\frac{2}{\cosh ^{2} x} .
$$

It turns out this potential possesses a remarkable property: it is reflectionless. In other words, it is absolutely transparent for incoming waves with any energy.

7 This potential was first considered in 1930 by Epstein [130] and Eckart [127], who pointed out its reflectionless character. 
In order to see it we can solve the eigenvalue problem directly by reducing it to the hypergeometric differential equation (see, e.g., [230]). However, for sake of diversity here we follow another route [183].

Note that (1.53) can be written as

$$
\hat{a}^{\dagger} \hat{a} \xi(x)=\omega^{2} \xi(x),
$$

where we introduced the ladder operators

$$
\hat{a}^{\dagger}=-\frac{d}{d x}+\tanh x ; \quad \hat{a}=\frac{d}{d x}+\tanh x .
$$

We define the ground state $\xi_{0}$ as the state annihilated by the operator $\hat{a}$, i.e.,

$$
\hat{a} \xi_{0} \equiv\left(\frac{d}{d x}+\tanh x\right) \xi(x)=0 .
$$

Up to a normalization factor, the solution to this equation is

$$
\xi_{0}(x)=\frac{1}{\cosh x} .
$$

This is the translation zero mode with zero eigenvalue $\omega_{0}=0$. Indeed, an excitation of this mode corresponds to the configuration

$$
\begin{aligned}
\phi_{K}(x) & \rightarrow \phi_{K}(x)+C \xi_{0}(x)=4 \arctan \left(e^{x}\right)+\frac{C}{\cosh x} \\
& =\phi_{K}(x)+\frac{C}{2} \frac{d \phi_{K}(x)}{d x} \approx \phi_{K}(x+C / 2),
\end{aligned}
$$

where $C$ is some number. Clearly, this is the kink solution translated by $\delta x=C / 2$. Of course, the energy of the configuration remains unchanged by this translation. On the other hand, appearance of the zero mode (1.58) in the spectrum of fluctuations reflects the translation invariance of the sine-Gordon model, which was violated by the localized field configuration (1.28).

We can now try to find another excited mode acting by the operator $\hat{a}^{\dagger}$ on the ground state. However there is no other discrete level in the problem with potential (1.53), thus only continuum modes appear in the spectrum.

Using the parametrization $\xi(x)=f(x) e^{i k x}$ we can find these modes as the solution of the corresponding differential equation on the function $f(x)$. After some algebra we get

$$
\xi_{k}(x)=(\tanh x+i k) e^{i k x} \quad \omega_{k}=\sqrt{1+k^{2}} .
$$

It is noticeable that

$$
\hat{a} e^{i k x} \equiv\left(\frac{d}{d x}+\tanh x\right) e^{i k x}=\xi_{k}(x) .
$$

This is the plane wave propagating along the profile of the kink (the meson). The mass of these excitations in dimensionless units is $m=1$. This result actually agrees with our evaluation of the interaction potential between the kinks (1.51). 
Indeed, this force is mediated by the Yukawa interaction, which, for the large separation $R=2 d$ between the solitions, is $e^{-m R}$. Thus, there is a gap in the energies $0<\omega<1$.

We can now find the asymptotic form of the excitations. In the remote past the modes (1.60) are infinitely far away from the kink, thus $\xi(-\infty)=(-1+i k) e^{i k x}$, while in the remote future they approach the asymptotic form $\xi(\infty)=(1+i k)$ $e^{i k x+\delta}$. Therefore, there is no reflected wave and the additional phase shift acquired by the incoming wave is

$$
e^{i \delta_{k}}=\frac{i k+1}{i k-1},
$$

while the phase shift of the transmitted wave relative to the incident wave is $\delta_{k}=\pi-\arctan \left(2 k /\left(k^{2}-1\right)\right)$.

Finally, note that there are no negative eigenmodes with $\omega^{2}<0$ in the spectrum of fluctuations, thus the kink configuration is stable.

\subsubsection{Breathers}

We mentioned already that the kink-antikink pair cannot annihilate into a trivial vacuum state. The reason for this unusual behavior is the integrability of the model, which has an infinite number of integrals of motion. However, this pair may form a bound state, which is another extremely interesting non-perturbative solution of the sine-Gordon equation. It describes the tightly bounded kink and antikink, which are coupled together and oscillating with a constant frequency. This solution is known as a breather (see Figure 1.7).

In order to obtain this solution explicitly, we have to perform the analytical continuation of the velocity parameter $v \rightarrow \frac{i \omega}{\sqrt{1-\omega^{2}}}$ in the kink-antikink solution (1.48). Then in the rest frame, we obtain

$$
\phi_{2} \equiv \phi_{\omega}=4 \tan ^{-1}\left(\frac{\sqrt{1-\omega^{2}}}{\omega} \frac{\sin \omega t}{\cosh \left(x \sqrt{1-\omega^{2}}\right)}\right) .
$$

By definition, the topological charge of this localized stable configuration is zero. However, similar to the $K \bar{K}$ system it cannot annihilate into a trivial vacuum. An evident similarity of this configuration with the kink-antikink part can be seen when we compare the Figure 1.5 and Figure 1.7, bottom panel. Since the frequency $\omega$ remains a free parameter, there is a family of the breather solitons interpolating between the small-amplitude quasi-phonon oscillations about the trivial vacuum and the kink-antikink pair. Note that the solution (1.62) corresponds to the stationary breather; evidently it can be boosted via the usual Lorentz transformation.

Substituting the breather solution (1.62) into general formula for the energy (1.15) after some calculations we get

$$
E_{\omega}=16 \sqrt{1-\omega^{2}}
$$



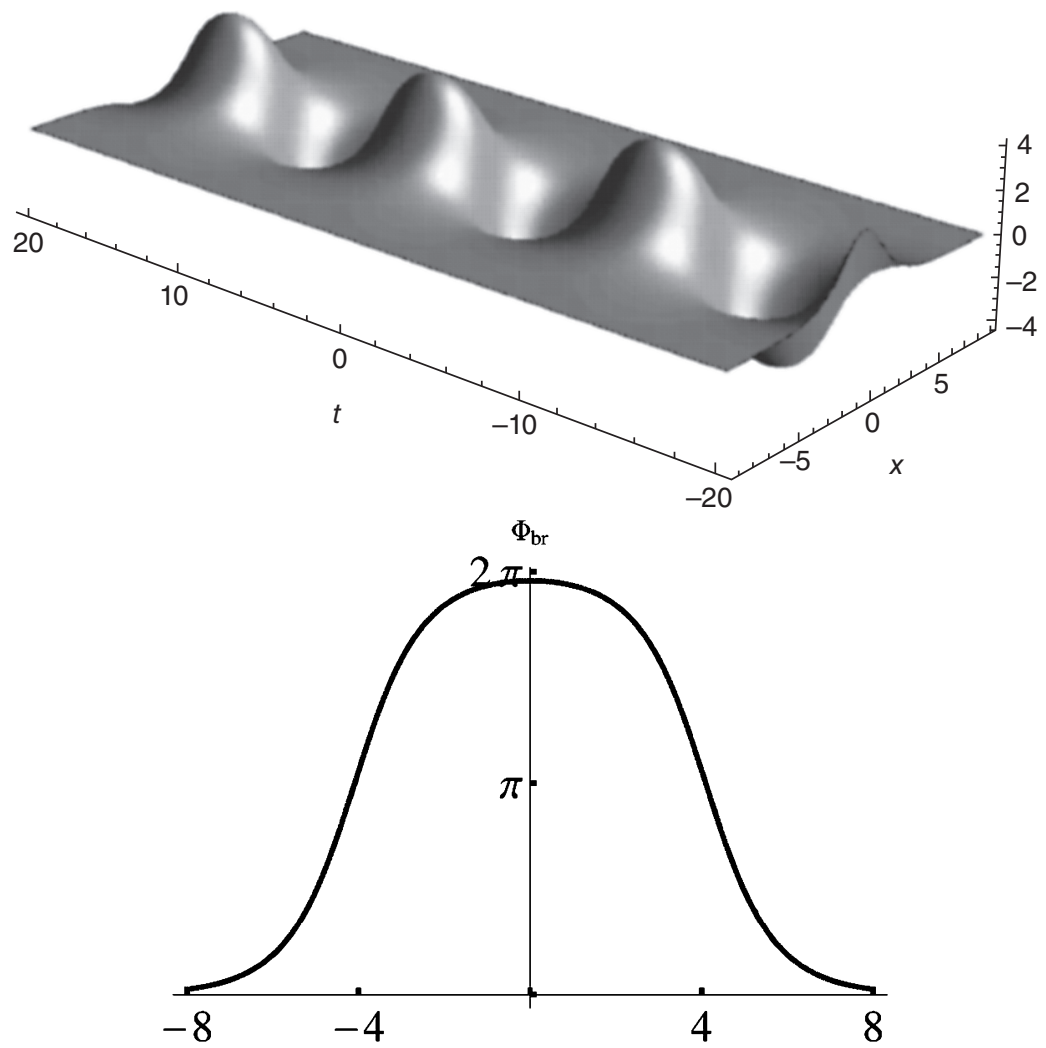

Figure 1.7 The field of the breather solution (1.62) as a function of variables $x, t$ at $\omega=0.5$ (upper plot) and the snapshot of the field of the breather at $\omega=0.001, t=30$ (bottom plot).

which is less than the energy of the kink-antikink pair for any nonzero values of $\omega$, thus the breather cannot decay.

Clearly, existence of the breathers sheds new light on the kink-antikink collision. We can consider it as a three-stage process. At the first step, the approaching solitons form an intermediate, excited breather state, energy of which is above the bound (1.63) for any nonzero impact velocity. Then this "quasi-breather" starts to oscillate and in the half period its field approaches the neighboring vacuum. Thus, at the final step the soliton-antisoliton pair is reborn.

Remember that the integrability of the model does not allow for any nontrivial processes. Even the collision between the kink and the breather is completely elastic. This composite configuration, which is another exact solution of the sineGordon equation (1.8), can be considered a superposition of the corresponding separated solitons moving toward the collision center. ${ }^{8}$ Numerical simulation

8 More precisely, such a configuration can be constructed by writing the three-soliton kink-antikink-kink solution of the sine-Gordon equation in Hirota's form [411] and consequent complex conjugation of the corresponding parameter. 


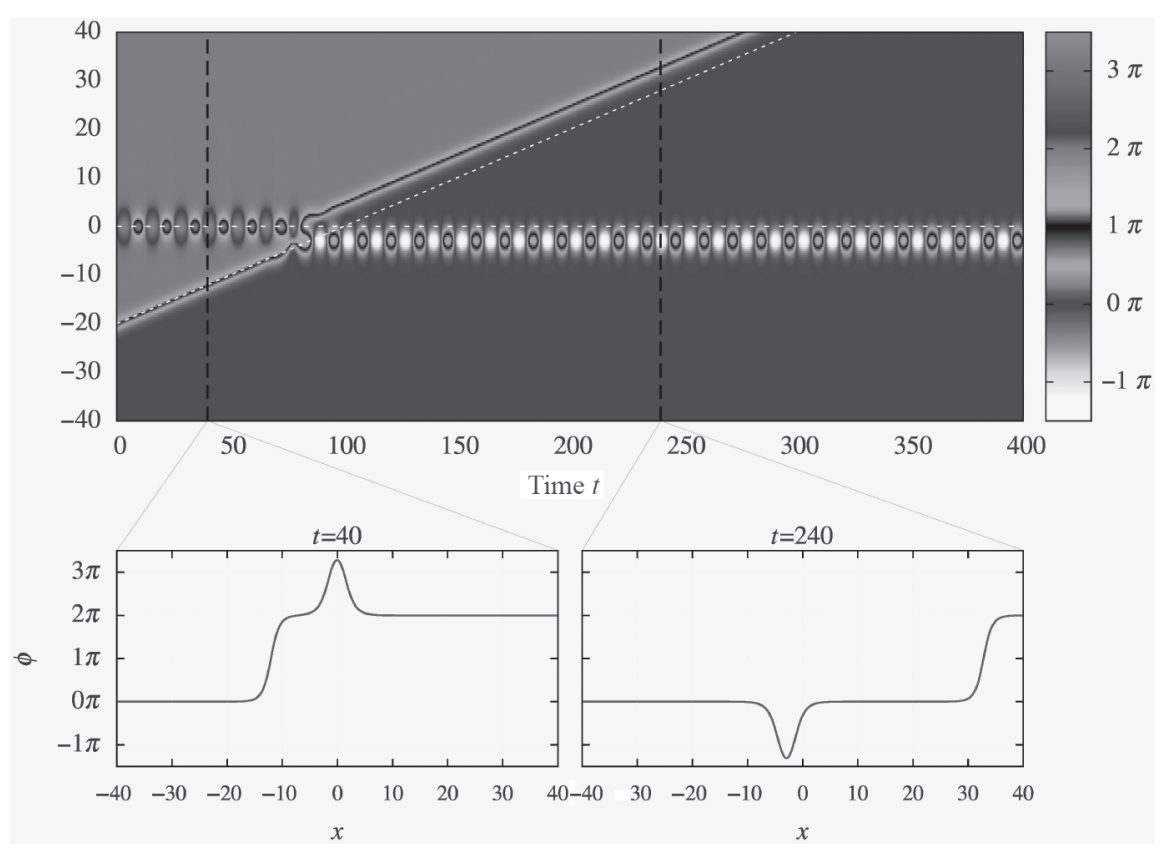

Figure 1.8 Scattering of the kink on the breather: Contour plots of the scalar field as a function of variables $x, t$ at $\omega=0.5$ (top panel) and the snapshots of the field of the kink-breather configurations at $t=50$ and $t=250$ (bottom panel)(Courtesy of Tomasz Romanczukiewicz).

shows that the outcome of the collision depends on the impact velocity. If the initial kinetic energy of the kink is smaller than the energy of the breather, the kink is elastically reflected from the breather with a small time delay in agreement with quantity $2 \delta$. If the kinetic energy of the kink exceeds the energy of the breather, the elastic scattering of the kink on the breather results in the passage of the kink through the breather with small displacement of the solitons by the same amount, $2 \delta$. The breather survives the passage being transferred from the top vacuum $2 \pi n$ to the bottom vacuum $2 \pi(n-1)$. In Figure 1.8 we display the results of direct numerical simulations and the profiles of the initial and final configurations.

Discussing collisions of the solitons, remember that they possess some internal structure and are not quite particle-like states. Particularly, at some moment in time the central collision between the kink and antikink through the quasibreather intermediate state instantaneously results in a trivial vacuum state when both solitons are losing their identity. Similarly, a collision between the kink and the breather, which actually represents a strongly coupled kink-antikink pair, may be treated as a process in which the incoming kink knocks out one of the bounded components and replaces it in the recreated breather. 


\subsection{Integrability: The Sine-Gordon Model}

The idea of classical integrability is related to nice properties of some Hamiltonian systems in that they may support an infinite tower of independent integrals of motion. More precisely, these systems are described by some set of nonlinear differential equations that - at least in principle - can be solved analytically, thus these systems are exactly solvable.

Unfortunately, there is no generic prescription that would allow us to identify the integrable systems at first glance. One needs to work out a specific approach to reveal integrability of each particular system under consideration. ${ }^{9}$

In this section, we discuss some general aspects of the concept of classical integrability that are relevant to our discussion. Actually, our goal is to provide an introduction, which is necessary to understand the role this beautiful idea is playing in the discussion of solitons. There are many good reviews on this subject that may give a thorough introduction to the basis of integrability (see, e.g., $[38,125]$ and references therein). Here, we only comment on the basics of integrability. In particular, for the sake of simplicity, we will not discuss here very a powerful and mathematically refined approach to integrable systems, the inverse scattering technique.

Given a Hamiltonian system, how does one find whether it is an integrable system or not? How does one find these independent conserving charges explicitly, especially taking into account that there any infinitely many of them?

Sadly, as mentioned above there is no general recipe; each particular system must be treated differently. As a first step, let us clarify the definition of an integral of motion. Recall that in a classical Hamiltonian theory a (local) conserved quantity $Q$ is defined as a function of configuration space that is invariant with respect to evolution in time, i.e.,

$$
\frac{d Q}{d t}=\{H, Q\}=0,
$$

where $H$ is the Hamiltonian of the system. In the field theory the Hamiltonian is defined as a functional over the infinite-dimensional space of field configurations. For example, in the sine-Gordon model we have (cf. (1.15))

$$
H[\phi]=\int_{-\infty}^{\infty} d x\left[\frac{1}{2} \Pi^{2}+\frac{1}{2}\left(\partial_{x} \phi\right)^{2}+(1-\cos \phi)\right],
$$

where the canonical momentum density is

$$
\Pi=\frac{\delta L}{\delta \dot{\phi}}=\partial_{t} \phi
$$

9 "Note that there is the Painlevé test of integrability of a system of dynamical equations [316], based on the study of corresponding critical points. However, this algorithm is mainly restricted to relatively simple low order systems in one spacial dimensions." 
The functional derivative, which arrears here, is defined as

$$
\frac{\delta L}{\delta \phi(x)}=\frac{\partial L}{\partial \phi}-\frac{\partial}{\partial x} \frac{\partial L}{\partial \phi_{x}}+\frac{\partial^{2}}{\partial x^{2}} \frac{\partial L}{\partial \phi_{x x}}+\ldots
$$

and

$$
\frac{\partial \phi(x)}{\partial \phi(y)}=\delta(x-y) .
$$

The generalization of the Poisson bracket of the functionals $H$ and $Q$, which preserves the antisymmetry and satisfies the Jacobi identity, is

$$
\{H, Q\}=\int_{-\infty}^{\infty} d x \frac{\delta H}{\delta \phi} \frac{\partial}{\partial x} \frac{\delta Q}{\delta \phi},
$$

and the time evolution of the system is defined by the Hamiltonian equations of motion

$$
\Pi=\partial_{t} \phi=\{\phi, H[\phi]\}=\frac{\partial}{\partial x} \frac{\delta H}{\delta \phi} .
$$

The usual Hamiltonian formalism for finite-dimensional systems can be recovered if we expand the field $\phi(x, t)$ in Fourier series, $\phi(x, t)=\sum f_{k} e^{i k x}$. Substituting this expansion into (1.69) we can see that the quantities $q_{k}=f_{k} / k, p_{k}=f_{-k}$ and $\mathcal{H}=\frac{i}{2 \pi} H$, for each Fourier mode satisfy the conventional equations

$$
\frac{d q_{k}}{d t}=\frac{\partial \mathcal{H}}{\partial p_{k}} ; \quad \frac{d p_{k}}{d t}=-\frac{\partial \mathcal{H}}{\partial q_{k}}
$$

and the Poisson bracket (1.68) becomes

$$
\{H, Q\}=\frac{i}{2 \pi} \sum_{k=-\infty}^{\infty} k \frac{\partial H}{\partial f_{k}} \frac{\partial Q}{\partial f_{-k}} .
$$

Evidently, the energy functional itself and the momentum $P(1.35)$ are the integrals of motion. However it is convenient to express these integrals via two conserved quantities

$$
\begin{aligned}
I_{ \pm 1} & =\int_{-\infty}^{\infty} d x\left[\frac{1}{2}\left(\partial_{t} \phi \pm \partial_{x} \phi\right)^{2}+(1-\cos \phi)\right] \\
& =\int_{-\infty}^{\infty} d x\left[2\left(\partial_{ \pm} \phi\right)^{2}+V(\phi)\right]
\end{aligned}
$$

thus, $H=\frac{1}{2}\left(I_{1}+I_{-1}\right)$ and $P=\frac{1}{2}\left(I_{1}-I_{-1}\right)$.

The form of this structure suggests the following algorithm of construction of integrals of motion. Let us consider two quantities $X, Y$, which are some functions of the field $\phi$ and its derivatives. Suppose they satisfy the equation $\partial_{t}(X-Y)=$ $\partial_{x}(X+Y)($ cf. (1.14)) and impose the condition that the combination $X+Y$ 
approaches the same finite value on both ends of the spacial line. Then, integrating this equation over the space and changing the order of differentiation and integration in the left-hand side, we get

$$
\frac{d}{d t} \int_{-\infty}^{\infty} d x(X-Y)=\left.(X+Y)\right|_{-\infty} ^{\infty}=0
$$

that is, the quantity $I=\int d x(X-Y)$ is conserved. Evidently, in our consideration, $X=2\left(\partial_{ \pm} \phi\right)^{2}$ and $Y=-V[\phi]$. Then we can continue our search for other conserved quantities considering polynomials of higher degrees in derivatives. For example, the next suitable pair of third degree is ${ }^{10}$

$$
I_{ \pm 3}=\int_{-\infty}^{\infty} d x\left[\frac{1}{2}\left(\partial_{ \pm}^{2} \phi\right)^{2}-\frac{1}{8}\left(\partial_{ \pm} \phi\right)^{4}+\left(\partial_{ \pm} \phi\right)^{2}(\cos \phi)\right] .
$$

We can check directly that this quantity commutes with the Hamiltonian (1.65). This procedure can be extended; however, it is a bit tedious to continue our search for corresponding polynomials of higher degrees. Evidently, there is a general formulation that allows us to find, at least in principle, all sets of conserved quantities.

Now we briefly describe how this goal can be achieved. It is convenient to start from the reformulation of the sine-Gordon model in terms of the Lax representation. The idea is that each integrable nonlinear system can be represented in the form of the compatibility condition between two linear equations of a Lax pair [264].

Let us consider two matrix-valued functions $A_{\rho}, A_{\tau}$ of the light-cone coordinates $\rho, \tau(1.36)$, which also depend on the auxiliary variable $\lambda \in \mathbb{C}$. This parameter is referred to as the spectral parameter. Suppose these matrices satisfy the system of two linear equations:

$$
\frac{\partial \psi}{\partial \rho}=A_{\rho} \psi ; \quad \frac{\partial \psi}{\partial \tau}=A_{\tau} \psi
$$

where $\psi(\rho, \tau)$ is an auxiliary column vector that is assumed to satisfy the compatibility condition similar to (1.40):

$$
\frac{\partial^{2} \psi}{\partial \rho \partial \tau}=\frac{\partial A_{\rho}}{\partial \tau} \psi+A_{\rho} \frac{\partial \psi}{\partial \tau} ; \quad \frac{\partial^{2} \psi}{\partial \tau \partial \rho}=\frac{\partial A_{\tau}}{\partial \rho} \psi+A_{\tau} \frac{\partial \psi}{\partial \rho} .
$$

Thus,

$$
\partial_{\tau} A_{\rho} \psi+A_{\rho} A_{\tau} \psi=\partial_{\rho} A_{\tau} \psi+A_{\tau} A_{\rho} \psi
$$

for any function $\psi(\rho, \tau)$. In other words, we have a zero curvature condition

$$
\partial_{\tau} A_{\rho}-\partial_{\rho} A_{\tau}=\left[A_{\tau}, A_{\rho}\right]
$$

10 There is no nontrivial solution of the second degree. 
This relation has a clear interpretation in terms of differential geometry, where the equations $\left(\partial_{\rho}-A_{\rho}\right) \psi=0$ and $\left(\partial_{\tau}-A_{\tau}\right) \psi=0$ describe the parallel transport of a vector $\psi$ in the $\rho$ and $\tau$ directions, respectively. The matrices $A_{\rho}$ and $A_{\tau}$ are referred to as the connection coefficients, thus the condition (1.77) defines a connection one-form on a two-dimensional vector bundle over the $\tau, \rho$ plane: $d A=A_{\rho} d \rho+A_{\tau} d \tau$. Then the zero curvature condition (1.77) means that the parallel transport of a vector $\psi$ from one point on the $\tau, \rho$ plane to another does not depend on the path connecting these points.

Let

$$
\begin{aligned}
& A_{\rho}=i \lambda\left(\begin{array}{cc}
1 & 0 \\
0 & -1
\end{array}\right)+\frac{i}{2}\left(\begin{array}{cc}
0 & \phi_{\rho} \\
\phi_{\rho} & 0
\end{array}\right)=i \lambda \cdot \sigma_{3}+\frac{i}{2} \phi_{\rho} \cdot \sigma_{1} \\
& A_{\tau}=\frac{\cos \phi}{4 i \lambda}\left(\begin{array}{cc}
1 & 0 \\
0 & -1
\end{array}\right)+\frac{\sin \phi}{4 i \lambda}\left(\begin{array}{cc}
0 & -i \\
i & 0
\end{array}\right)=\frac{\cos \phi}{4 i \lambda} \cdot \sigma_{3}+\frac{\sin \phi}{4 i \lambda} \cdot \sigma_{2},
\end{aligned}
$$

where $\sigma_{i}$ are the usual traceless, Hermitian Pauli matrices. Hence, the $2 \times 2$ connection matrices $A_{\rho}, A_{\tau}$ are taking values in the Lie algebra $s u(2)$.

The zero curvature condition (1.77) then becomes

$$
\frac{1}{2 i}\left(\sin \phi-\partial_{\rho \tau} \phi\right) \cdot \sigma_{1}=0
$$

i.e., it is equivalent to the sine-Gordon equation on the function $\phi(\rho, \tau)[2]$. Evidently, this correspondence holds for any value of the spectral parameter $\lambda$.

Since the matrices $A_{\rho}, A_{\tau}$ are in the Lie algebra $s u(2)$, there is a freedom in their definition, which is related to the usual gauge transformations

$$
\begin{aligned}
& A_{\rho} \rightarrow \tilde{A}_{\rho}=U A_{\rho} U^{-1}+\partial_{\rho} U U^{-1} \\
& A_{\tau} \rightarrow \tilde{A}_{\tau}=U A_{\tau} U^{-1}+\partial_{\tau} U U^{-1},
\end{aligned}
$$

where $U(\rho, \tau)$ is an arbitrary invertible $S U(2)$ matrix. This transformation actually defines a symmetry of the equation of motion (1.8); it is an underlying reason for the existence of an infinite set of integrals of motion in the sine-Gordon system.

Note that, in a general case, in order to recover the original sine-Gordon equation from the zero curvature condition imposed on the matrices $\tilde{A}_{\rho}, \tilde{A}_{\tau}$, we have to separate out the coefficients of powers of $\lambda$ in the resulting matrixvalued equation. Thus the gauge (1.78) is particularly convenient in the light-cone coordinates $\rho, \tau$.

Obviously, the Lax formulation does not depend on a particular choice of the coordinates. In particular we can return to the usual coordinates $(x, t)$ and instead of the Lax pair (1.78), consider another $2 \times 2$ matrices

$$
\begin{aligned}
A_{x} & =\frac{i}{4}\left(\begin{array}{cc}
\partial_{t} \phi & 2 \sinh (a+i \phi / 2) \\
2 \sinh (a-i \phi / 2) & -\partial_{t} \phi
\end{array}\right) ; \\
A_{t} & =\frac{i}{4}\left(\begin{array}{cc}
\partial_{x} \phi & -2 \cosh (a+i \phi / 2) \\
-2 \cosh (a-i \phi / 2) & -\partial_{x} \phi
\end{array}\right) .
\end{aligned}
$$


Further, these matrices can be expanded in the $s u(2)$ basis as

$$
\begin{aligned}
& A_{x}=\frac{i}{4}\left(\lambda-\frac{1}{\lambda}\right) \cos \phi \cdot \sigma_{1}-\frac{i}{4}\left(\lambda+\frac{1}{\lambda}\right) \sin \phi \cdot \sigma_{2}+\frac{i}{4} \partial_{t} \phi \cdot \sigma_{3} \\
& A_{t}=-\frac{i}{4}\left(\lambda+\frac{1}{\lambda}\right) \cos \phi \cdot \sigma_{1}+\frac{i}{4}\left(\lambda-\frac{1}{\lambda}\right) \sin \phi \cdot \sigma_{2}+\frac{i}{4} \partial_{x} \phi \cdot \sigma_{3},
\end{aligned}
$$

where we restore the conventional spectral parameter $\lambda=e^{a}$. Then the zero curvature condition in the $(x, t)$ variables after some straightforward calculations yields

$$
\begin{aligned}
{\left[\partial_{x}-A_{x}, \partial_{t}-A_{t}\right] } & \equiv \partial_{t} A_{x}-\partial_{x} A_{t}+\left[A_{x}, A_{t}\right] \\
& =\frac{i}{4}\left(\sin \phi+\partial_{t t} \phi-\partial_{x x} \phi\right) \cdot \sigma_{3}=0 .
\end{aligned}
$$

Thus, we recover the original sine-Gordon equation (1.8) again.

Let us consider the zero curvature condition in more detail. The covariant derivative $D_{x}=\partial_{x}-A_{x}$ corresponds to the parallel transport of a vector $\psi$ in $x$-direction. We can describe it in terms of the space-time dependent transfer matrix $\psi^{\prime}=T \psi$, where the transported vector $\psi^{\prime}$ satisfies the equation $D_{x} \psi^{\prime} \equiv$ $\left(\partial_{x}-A_{x}\right) \psi^{\prime}=0$, i.e.,

$$
\left(\partial_{x}-A_{x}\right) T(x, y, t)=0,
$$

with the initial condition $T(x, x)=\mathbb{I}$. This equation has a general solution written as a path-ordered exponent

$$
T\left(x_{1}, x_{2}, t\right)=\mathcal{P} \exp \left[\int_{x_{1}}^{x_{2}} d x A_{x}(x, t)\right] .
$$

Similarly, a translation in $t$-direction is given by the exponent

$$
S\left(t_{1}, t_{2}, x\right)=\mathcal{P} \exp \left[\int_{t_{1}}^{t_{2}} d t A_{t}(x, t)\right] .
$$

Then the zero curvature condition, which is also the condition of commutativity of covariant derivatives, means that a parallel transport along a closed contour gives the identity: ${ }^{11}$

$$
W=\mathcal{P} \exp \left[\oint d x^{\mu} A_{\mu}(x, t)\right]=\mathbb{I} .
$$

11 In quantum field theory such a contour is known as the Wilson loop. This gauge-invariant observable plays a central role in the lattice formulation of the gauge theory (see, e.g., $[112])$. 
Indeed, let us introduce the monodromy matrix $L(t)=T(-\infty, \infty, t)$ and consider a closed path in the $x, t$ plane. Due to the flatness condition, the Wilson loop is invariant under continuous deformations of the path contour. In particular, we can consider a rectangular path along the contour, which starts at $x=-\infty$ at $t=t_{1}$, and then consequently goes through the points $\left(\infty, t_{1}\right),\left(\infty, t_{2}\right)$, $\left(-\infty, t_{2}\right)$, and back to $\left(-\infty, t_{1}\right) \cdot{ }^{12}$ Further, taking into account the explicit form of the Lax pair (1.81) and asymptotic behavior of the field $\phi(x)$, which yields $A_{t}(\infty, t)=A_{t}(-\infty, t)$, we can define the asymptotic matrix

$$
M \equiv A_{t}(\infty)=-\frac{i}{4}\left(\lambda-\frac{1}{\lambda}\right) \cdot \sigma_{1} .
$$

For such a contour we have the Wilson loop composed of four transfer matrices as

$$
\begin{aligned}
& S^{-1}\left(t_{1}, t_{2}, \infty\right) L^{-1}\left(t_{2}\right) S\left(t_{1}, t_{2}, \infty\right) L\left(t_{1}\right) \\
& \quad=e^{\left(t_{1}-t_{2}\right) M} L^{-1}\left(t_{2}\right) e^{\left(t_{2}-t_{1}\right) M} L\left(t_{1}\right)=\mathbb{I} .
\end{aligned}
$$

This formula can be rewritten as

$$
e^{\left(t_{2}-t_{1}\right) M} L\left(t_{1} ; \lambda\right) e^{\left(t_{1}-t_{2}\right) M}=L\left(t_{2} ; \lambda\right) .
$$

Evidently, when we consider an infinitesimally small time interval $\delta t=t_{2}-t_{1} \rightarrow 0$ this relation yields the Lax equation

$$
\frac{d L(\lambda)}{d t}=[M(\lambda), L(\lambda)]
$$

Therefore the trace of the matrix $W(\lambda)$ does not depend on time. By construction we obtain the set of integrals of motion, for example,

$$
\frac{d \operatorname{Tr}\left(L^{n}\right)}{d t}=n \operatorname{Tr}\left(L^{n-1} \dot{L}\right)=n \operatorname{Tr}\left(L^{n-1}[M, L]\right)=0
$$

for an arbitrary $n$.

More generally, expanding the trace $\operatorname{Tr} L(\lambda)$ in powers of $\lambda$ or $\lambda^{-1}$ we can now get an infinite number of conserved quantities that are all coefficients of the corresponding Laurent series expansion.

Explicitly, we have to make use of the gauge freedom to cast the Lax conection in the diagonal form (see, e.g., [38]). First, we make use of the gauge transformation (1.79) with the matrix $e^{\frac{i}{4} \phi \cdot \sigma_{3}}$. Then the matrix of the Lax connection $A_{x}$ defined by (1.81) transforms to

$$
A_{x} \rightarrow \tilde{A}_{x}=\frac{i}{4}\left[\left(\lambda \cos \phi-\frac{1}{\lambda}\right) \cdot \sigma_{1}-\lambda \sin \phi \cdot \sigma_{2}+\left(\partial_{t} \phi+\partial_{x} \phi\right) \cdot \sigma_{3}\right] .
$$

12 This procedure becomes much more simple in the sine-Gordon model with periodic boundary conditions. 
The connection $\tilde{A}_{x}$ lies in the $s u(2)$ algebra, $\tilde{A}_{x}=A_{x}^{a} \cdot \sigma^{a}$, thus it is always possible to transform it to the Abelian gauge by means of a suitable rotation

$$
U\left(A_{x}^{a} \cdot \sigma^{a}\right) U^{-1}+\partial_{x} U U^{-1}=f(x) \cdot \sigma_{3},
$$

Then the transformed monodromy matrix becomes diagonal, i.e.,

$$
L \rightarrow \tilde{L}=\exp \left[\int_{-\infty}^{\infty} d x f(x) \sigma_{3}\right]
$$

where the function $f(x)$ can be found from the corresponding equation of Ricatti type. In other words, the transformed monodromy matrix is now given by a plain integral without path ordering. Finally, the conserved charges $I_{+n}$ are the coefficients in the expansion of $\operatorname{Tr}(L(\lambda))=2 \cos \int d x f(x)$ in powers of $\lambda^{-1}$. The complementary set of charges $I_{-n}$ can be constructed via the conjugated transformation $e^{-\frac{i}{4} \phi \cdot \sigma_{3}}$ and consequent diagonalization of the Lax connection.

\subsection{1 $\mathbb{C P}^{1}$ Sine-Gordon Model}

An interesting generalization of the sine-Gordon model is related to mapping of the scalar field $\phi(x, t)$ onto the complex-valued massive field $w=w_{1}+i w_{2}$, which is a function of the space-time coordinates, i.e., it is a map from $\mathbb{R}^{1+1}$ to $\mathbb{C P}^{1} \cong S^{2}$. We consider the following Lagrangian

$$
L=\frac{\partial_{\mu} w \partial^{\mu} \bar{w}-m^{2}|w|^{2}}{\left(1+|w|^{2}\right)^{2}},
$$

which defines the $\mathbb{C P}^{1}$ model with a mass term. This theory was suggested by Lund and Regge to model dynamics of vortices in a superfluid in an external field [278]. Note that in general we do not restrict the field $w$ to the surface of unit sphere, though the scale invariance of the $\mathbb{C P}^{1}$ model always makes it possible.

The energy density of the model is

$$
E=\frac{\left|\partial_{t} w\right|^{2}+\left|\partial_{x} w\right|^{2}+m^{2}|w|^{2}}{\left(1+|w|^{2}\right)^{2}} .
$$

Let us consider the case $m^{2} \neq 0$. There are two vacua that correspond to $w=0$ and $w= \pm \infty$, thus one can expect there to be a soliton solution that interpolates between these vacua.

Clearly, the model is invariant with respect to $U(1)$ transformations $w \rightarrow e^{i \delta} w$, thus the corresponding conserving Noether current is

$$
J_{\mu}=\frac{i\left(w \partial_{\mu} \bar{w}-\bar{w} \partial_{\mu} w\right)}{\left(1+|w|^{2}\right)^{2}}
$$


and there is a conserved quantity

$$
Q=i \int_{-\infty}^{\infty} d x \frac{w \partial_{0} \bar{w}-\bar{w} \partial_{0} w}{\left(1+|w|^{2}\right)^{2}}
$$

The relation of this model with the sine-Gordon theory can be set if we map the field $w$ to the circle via stereographic projection

$$
w=\tan \left(\frac{\phi}{4}\right) e^{i \theta}
$$

where $\phi$ and $\theta$ are angular parameters on the sphere $S^{2}$. Then the Lagrangian (1.91) becomes

$$
L=\frac{1}{16} \partial_{\mu} \phi \partial^{\mu} \phi-\frac{1}{4}\left(m^{2}-\partial_{\mu} \theta \partial^{\mu} \theta\right) \sin ^{2} \frac{\phi}{2}
$$

In other words, we arrive at the model that describes two nonlinearly coupled real scalar fields, $\phi(x, t)$ and $\theta(x, t)$, and the mass of field $\phi$ depends on the derivatives of the massless component $\theta(x, t)$.

Evidently, if the effective mass term is positive, i.e., for $m^{2}>\partial_{\mu} \theta \partial^{\mu} \theta$, the model has an infinite number of degenerate vacua at $\phi_{0}=2 \pi n, \quad n \in \mathbb{Z}$ is similar to the sine-Gordon model (1.9).

The analogue of the expression for the energy bound (1.21) can be easily written in the form

$$
\begin{aligned}
E= & \frac{1}{16} \int_{-\infty}^{\infty} d x\left[\left(\partial_{t} \phi\right)^{2}+\left(\partial_{x} \phi-2 \sqrt{m^{2}+\left(\partial_{t} \theta\right)^{2}+\left(\partial_{x} \theta\right)^{2}} \sin \frac{\phi}{2}\right)^{2}\right. \\
& \left.+4\left(\partial_{x} \phi\right) \sqrt{m^{2}+\left(\partial_{t} \theta\right)^{2}+\left(\partial_{x} \theta\right)^{2}} \sin \frac{\phi}{2}\right] \\
\geqslant & \frac{m}{4} \int_{-\infty}^{\infty} d x\left(\partial_{x} \phi\right) \sin \frac{\phi}{2}=\frac{m}{4} \int d \phi \sin \frac{\phi}{2} .
\end{aligned}
$$

One can see that the energy is minimal for the static configurations with a spacial independent component $\theta$, i.e., if $\partial_{t} \phi=\partial_{t} \theta=\partial_{x} \theta=0$. Then the bound is attained if

$$
\frac{\partial \phi}{\partial x}= \pm 2 m \sin \frac{\phi}{2}
$$

which is just the Bogomolny bound (1.26) with explicit mass parameter $m$. Clearly, the solution is the kink $(1.28) \phi(x)=4 \arctan e^{\sqrt{m^{2}}\left(x-x_{0}\right)}$ or

$$
w(x)=e^{\sqrt{m^{2}}\left(x-x_{0}\right)} .
$$


In this case the $U(1)$ charge of the configuration (1.94) is zero. Note that the normalization of the model is taken in such a way that the mass of the static kink is equal to one.

There is another interesting solution of the model (1.95), the Q-kink [202, 393]. In order to construct this solution we consider time-periodic component $\theta(t)=\omega t$. Then, for the static field $\phi(x)$ the minimal energy configuration is given by the oscillating field

$$
w(x, t)=e^{i \omega t} e^{\sqrt{m^{2}-\omega^{2}}\left(x-x_{0}\right)} .
$$

The name Q-kink appears because the oscillating solution carries the $U(1)$ charge. Indeed, one can see that the stationary rotations of the field $w(x, t)$ with a constant frequency $\omega$ yields the conserved charge

$$
Q=\frac{w}{2} \int_{-\infty}^{\infty} d x \sin ^{2} \frac{\phi}{2}=\frac{\omega}{\sqrt{m^{2}-\omega^{2}}},
$$

thus, the model admits spinning kink solutions, which are also referred to as Q-kinks [268, 393]. In Chapter 6 we consider similar charged localized field configurations, both in $1+1$ dimensions and in higher-dimensional models, so we will not discuss here the details of Q-kinks. Just note that the energy of the spinning configuration is

$$
E=\frac{m^{2}}{\sqrt{m^{2}-\omega^{2}}} .
$$

Hence the solution becomes unstable as $\omega>m$. In such a case it decays into the burst of radiation of scalar quanta. 